\title{
LYAPUNOV SPECTRA FOR KAPITZA OSCILLATOR
}

\author{
Nayyer IqBal ${ }^{1}$, Shahid Ahmad ${ }^{2}$ And Muhammad Hussain ${ }^{3}$ \\ ${ }^{1}$ Department of Mathematics, GC University, Lahore, Pakistan, \\ nayyersms3@gmail.com \\ ${ }^{2}$ Department of Mathematics, GC University, Lahore, Pakistan, \\ shahidsms@gmail.com \\ ${ }^{3}$ Department of Mathematics, COMSATS Institute of Information \\ Technology, Defence Road off Raiwind Road, Lahore, Pakistan, \\ mhmaths@yahoo.com
}

\begin{abstract}
Here we purpose a simple but realistic model of one dimensional nonlinear Kapitza oscillator driven by sin- or cos- rapidly external oscillating periodical force. The model has a parameter $2 g l / a^{2} \gamma^{2}$ of dimension one, depending on the amplitude $a$ and frequency of modulation $\gamma$. Changing its value we construct phase portraits of the system in the neighbourhood of fixed points and demonstrate the changing in Lyapunov spectrum. Our purpose is to observe the behavior of system at fixed points due to the different structures of the Lyapunov spectra.
\end{abstract}

Key words and Phrases: Kapitza oscillator, Lyapunov spectrum, Lyapunov spectra.

\begin{abstract}
Abstrak. Pada paper ini disajikan sebuah model sederhana tapi realistis dari osilator Kapitza nonlinear dimensi satu yang dikendalikan oleh gaya luar berosilasi periodik sin- dan cos- dengan cepat. Model ini mempunyai parameter $2 g l / a^{2} \gamma^{2}$ satu dimensi, bergantung pada amplitudo $a$ dan frekuensi modulasi $\gamma$. Dengan merubah nilai parameternya, kami mengkonstruksi diagram fasa dari sistim pada ketetanggaan titik tetap dan mendemonstrasikan perubahan spektrum Lyapunov. Kemudian, kami menyajikan pengamatan dari tingkah laku sistim pada titik tetap dikaitkan dengan struktur dari spektral Lyapunov.
\end{abstract}

Kata kunci: Osilator Kapitza, spektrum Lyapunov, spektral Lyapunov.

2000 Mathematics Subject Classification: 35P30

Received: 28-10-2010, revised: 07-06-2011, accepted: 08-06-2011. 


\section{Introduction}

An ordinary rigid planar pendulum suspended in the uniform gravitational field is a very useful and versatile physical model famous first of all for its outstanding role in the history of physics. The pendulum is also interesting as a paradigm of contemporary nonlinear physics and more importantly, because the differential equation of the pendulum is frequently encountered in various branches of modern physics. For example, the mathematical relationship associated with the limiting motion of a frictionless pendulum, play an important role in the theory of solitons, in the problem of supper-radiation in quantum optics, and in theory of Josephson effects in weak superconductivity. Kapitza oscillator is a simple pendulum with pivot oscillating with a very high frequency of external periodical force. It was named after Russian nobel laureate physicits " Pyotr Kapitza " which successfully explains some of its unusual properties in 1951. The unique feature of Kapitza pendulum is that the vibrating suspension can cause it to balance stably in an inverted position, with the bob above the suspension point and can be employed to different problems in applied sciences. For instance, in Quantum Mechanics, the trapping of particles [1-3], or in Mechanical engineering, the control of robotic devices [4,5]. Kapitza oscillator has different structure of its stable points under the influence of rapidly oscillating periodical force. The most traditional way to find out the stable points, is the method of effective potential energy proposed by Kapitza himself [6]. In this method we present the coordinate $x$ of the particle as a superposition with of the smooth movement $X$ and the rapidly changing part $\xi$

$$
x=X+\xi
$$

and then apply the averaging procedure with respect to the period $\mathrm{T}$ of the $\xi$ coordinate changing. We denote here this averaging by $\langle\ldots\rangle$, and we demand $<\xi>=0$. As a result the final dynamical equation doesn't depend on $\xi$ and it can be presented in the form

$$
m \ddot{X}=-\frac{U_{e f f}(X)}{d X}
$$

where the effective potential $U_{\text {eff }}$ comes from the averaging: $U_{\text {eff }}=U+$ (averaging terms from the external force $f$ ), and it is a function of the smooth coordinate $X$ only (for detail see [6]). The stable points can be easily found as minima of the effective potential $U_{\text {eff }}$. From the mathematical point of view, most of the works study linearized problem ([7][8]).In this paper we find stable points by the method of linearization and investigate the behavior of system by calculating the Lyapunov spectra. We investigate the Lyapunov spectra of the system in section 2. Table of results is shown in section 3 and support our analytical research by numerical simulations in section 4 . 


\section{Lyapunov exponents for Kapitza oscillator}

2.1. Motion of the pendulum whose point of support oscillates horizontally. Consider the motion of a pendulum of mass $m$ whose point of support oscillates horizontally in the plane of motion of the pendulum according to the law $x=a \cos \gamma t$ with a high frequency $\gamma>>\sqrt{\frac{g}{l}}$, where $g$ is the gravitational acceleration and $l$ is the length of pendulum. Now the differential equation of such a dynamical system is

$$
m l \ddot{\varphi}=-\frac{1}{l} \frac{d U_{e f f}}{d \varphi}
$$

where $\varphi=\varphi(t)$ is the angular displacement of the pendulum. From the Lagrangian derived in [9], we see in this case the variable force is

$$
f=m l a \gamma^{2} \cos \gamma t
$$

and

$$
U_{e f f}=m g l\left[-\cos \varphi+\left(\frac{a^{2} \gamma^{2}}{4 g l}\right) \cos ^{2} \varphi\right]
$$

is the effective potential energy, where $a$ is the amplitude of the oscillation. The dynamical system for horizontal modulation is

$$
m \ell^{2} \ddot{\phi}+m g \ell \sin \phi=\frac{1}{2} m a^{2} \gamma^{2} \sin \phi \cos \phi
$$

Now equation (4) in Cauchy form can be written as

$$
\left\{\begin{array}{l}
\dot{x_{1}}=x_{2} \\
\dot{x_{2}}=\frac{g}{\ell} \sin x_{1}\left(\frac{a^{2} \gamma^{2}}{2 g \ell} \cos x_{1}-1\right),
\end{array}\right.
$$

The solutions of the system (5) define the paths (phase trajectories) associated with the dynamical equation (4) in the $x_{1} x_{2}$ phase plane.Now we observe that fixed points of the system (4) are

$$
(k \pi, 0),\left(\arccos \left(\frac{a^{2} \gamma^{2}}{2 g \ell}\right)+2 k \pi, 0\right), k \in Z
$$

The linear terms often dominate near the fixed point, and in the case, they determine the behavior of the nonlinear equations near the fixed point; in particular, the linearized system (5) usually determines the stability of the fixed point.Here we calculate the Lyapunov exponents which distinguish chaotic and nonchaotic motions and are quantitative measures of the evolution of neighboring phase trajectories (for detail see [10]).Lyapunov exponents generalize the concept of eigenvalues of a fixed point.

Definition 2.1. If $x^{*}$ is a fixed point of $\dot{x}=F(x)$, then the eigenvalues of the matrix of partial derivatives $D F_{\left(x^{*}\right)}$ are the eigenvalues of the fixed point or the eigenvalues of $x^{*}$. 
Definition 2.2. A fixed point $x^{*}$ is called hyperbolic provided that the real parts of all the eigenvalues of the matrix $D F_{\left(x^{*}\right)}$ are nonzero.

If a fixed point is hyperbolic, then the stability type of the fixed point for the nonlinear system is the same as that of the linearized system. The following theorem states this more precisely.

Theorem 2.3. Consider a differential equation $\dot{x}=F(x)$ in $n$ variables, with a hyperbolic fixed point $x^{*}$. Assume that $F, \frac{\partial^{2} F_{i}}{\partial x_{j}}(x)$ and $\frac{\partial^{2} F_{i}}{\partial x_{j} \partial x_{k}}(x)$ are all continuous. Then, the stability type of the fixed point for the nonlinear system is the same as that for the linearized system at that fixed point.

(a) In particular if the real parts of all the eigenvalues of $D F_{\left(x^{*}\right)}$ are negative then the fixed point is asymptotically stable for the nonlinear equation (i.e., if the origin is asymptotically stable for the linearized system, then $x^{*}$ is asymptotically stable for the nonlinear equation ).

(b) If at least one eigenvalue of $D F_{\left(x^{*}\right)}$ has a positive real part, then the fixed point $x^{*}$ is unstable for the nonlinear equation. (The linearized system can be a saddle, unstable node, unstable focus, etc).

(c) If one of the eigenvalues of $D F_{\left(x^{*}\right)}$ has a zero real part, then the situation is more delicate. In particular, for $n=2$, if the fixed point is an elliptic center (eigenvalues $\pm \imath \beta$ ) or one eigenvalues is 0 of multiplicity one, then the linearized system does not determine the stability type of the fixed point [11].

Theorem 2.4. Assume that $x_{0}$ is a fixed point of the differential equation. Then, the Lyapunov exponents at the fixed point are the real parts of the eigenvalues of the fixed point [11].

We investigate the linearized stability of fixed points and calculate Lyapunov spectra at fixed points for horizontal modulation of the dynamical system (4). Eigenvalues at fixed point $(0,0)$ are

$$
\lambda= \pm \frac{a \gamma}{\sqrt{2} \ell} \sqrt{1-\frac{2 g \ell}{a^{2} \gamma^{2}}}
$$

By deffinition $(2)$, the fixed point $(0,0)$ is hyperbolic. Now since the eigenvalues are real, unequal, and of opposite signs so the origin is a saddle point and therefore the fixed point is unstable for nonlinear equation (4).

If $\frac{2 g \ell}{a^{2} \gamma^{2}}<1$ then Lyapunov spectrum is $\{+,-\}$

If $\frac{2 g \ell}{a^{2} \gamma^{2}} \geq 1$ then Lyapunov spectrum is $\{0,0\}$

Eigenvalues at fixed point $(\pi, 0)$ are

$$
\lambda= \pm \frac{a \gamma}{\sqrt{2} \ell} \sqrt{1+\frac{2 g \ell}{a^{2} \gamma^{2}}}
$$


If $\frac{2 g \ell}{a^{2} \gamma^{2}} \lesseqgtr 1$ then Lyapunov spectrum is $\{+,-\}$

Eigenvalues at fixed point $\left(\arccos \left(\frac{2 g \ell}{a^{2} \gamma^{2}}\right), 0\right)$ are

$$
\lambda= \pm \frac{a \gamma}{\sqrt{2} \ell} \sqrt{\left(\frac{2 g \ell}{a^{2} \gamma^{2}}\right)^{2}-1}
$$

If $\frac{2 g \ell}{a^{2} \gamma^{2}} \leq 1$ then Lyapunov spectrum is $\{0,0\}$

2.2. Motion of the pendulum whose point of support oscillates vertically. In this case the point of support of pendulum oscillates vertically according to the law $y=a \cos \gamma t$. From the Lagrangian derived in [9], we find

$$
f=-m l a \gamma^{2} \cos \gamma t
$$

and

$$
U_{e f f}=m g l\left[-\cos \varphi+\left(\frac{a^{2} \gamma^{2}}{4 g l}\right) \sin ^{2} \varphi\right]
$$

is the effective potential energy, where $a$ is the amplitude of the oscillation. The dynamical system for vertical modulation is

$$
m \ell^{2} \ddot{\phi}+m g \ell \sin \phi+\frac{1}{2} m a^{2} \gamma^{2} \sin \phi \cos \phi=0
$$

Equation (6) in Cauchy form can be written as

$$
\left\{\begin{array}{l}
\dot{x_{1}}=x_{2} \\
\dot{x_{2}}=-\frac{g}{\ell} \sin x_{1}\left(1+\frac{a^{2} \gamma^{2}}{2 g \ell} \cos x_{1}\right),
\end{array}\right.
$$

The fixed points of the system (7) are

$$
(k \pi, 0),\left(\arccos \left(-\frac{a^{2} \gamma^{2}}{2 g \ell}\right)+2 k \pi, 0\right), k \in Z
$$

Now we calculate Lyapunov spectra at fixed points for vertical modulation of the dynamical system (6).

Eigenvalues at fixed point $(0,0)$ are

$$
\lambda= \pm \frac{a \gamma}{\sqrt{2} \ell} \sqrt{1+\frac{2 g \ell}{a^{2} \gamma^{2}} i}
$$

Since eigenvalues are pure imaginary so in this case the fixed point is a center i.e we get rotation in the neighbourhood of origin and nonlinear system (6) is stable, but not asymptotically stable.

If $\frac{2 g \ell}{a^{2} \gamma^{2}} \lesseqgtr 1$ then Lyapunov spectrum is $\{0,0\}$

Eigenvalues at fixed point $(\pi, 0)$ are

$$
\lambda= \pm \frac{a \gamma}{\sqrt{2} \ell} \sqrt{\frac{2 g \ell}{a^{2} \gamma^{2}}-1}
$$


If $\frac{2 g \ell}{a^{2} \gamma^{2}} \leq 1$ then Lyapunov spectrum is $\{0,0\}$

If $\frac{2 g \ell}{a^{2} \gamma^{2}}>1$ then Lyapunov spectrum is $\{+,-\}$

Eigenvalues at fixed point $\left(\arccos \left(-\frac{2 g \ell}{a^{2} \gamma^{2}}\right), 0\right)$ are

$$
\lambda= \pm \frac{a \gamma}{\sqrt{2} \ell} \sqrt{1-\left(\frac{2 g \ell}{a^{2} \gamma^{2}}\right)^{2}}
$$

If $\frac{2 g \ell}{a^{2} \gamma^{2}}<1$ then Lyapunov spectrum is $\{+,-\}$

If $\frac{2 g \ell}{a^{2} \gamma^{2}}=1$ then Lyapunov spectrum is $\{0,0\}$

2.3. Motion of the pendulum whose point of support moves uniformly on a vertical circle. In this case point of support of the pendulum moves uniformly on a vertical circle with constant frequency $\gamma$. From the Lagrangian derived in $[9]$, the force $f$ is given by

$$
f=m l a \gamma^{2} \sin (\phi-\gamma t)
$$

and

$$
U_{\text {eff }}=m g l\left[-\cos \varphi+\left(\frac{a^{2} \gamma^{2}}{4 g l}\right)\right]
$$

is the effective potential energy, where $a$ is the amplitude of the oscillation. The dynamical system for vertical modulation is

$$
m \ell^{2} \ddot{\phi}+m g \ell \sin \phi=0
$$

Equation (8) in Cauchy form can be written as

$$
\left\{\begin{array}{l}
\dot{x_{1}}=x_{2} \\
\dot{x_{2}}=-\frac{g}{\ell} \sin x_{1},
\end{array}\right.
$$

The fixed points of the system (9) are

$$
(k \pi, 0), k \in Z
$$

We calculate Lyapunov spectra at fixed points for circulation modulation of the dynamical system (8). Eigenvalues at fixed point $(0,0)$ are

$$
\lambda= \pm \frac{a \gamma}{\sqrt{2} \ell} \sqrt{\frac{2 g \ell}{a^{2} \gamma^{2}}} i
$$

Since eigenvalues are pure imaginary so in this case the fixed point is a center i.e we get rotation in the neighbourhood of origin and nonlinear system (8) is stable, but not asymptotically stable.

If $\frac{2 g \ell}{a^{2} \gamma^{2}} \lesseqgtr 1$ then Lyapunov spectrum is $\{0,0\}$

Eigenvalues at fixed point $(\pi, 0)$ are

$$
\lambda= \pm \frac{a \gamma}{\sqrt{2} \ell} \sqrt{\frac{2 g \ell}{a^{2} \gamma^{2}}}
$$

If $\frac{2 g \ell}{a^{2} \gamma^{2}} \lesseqgtr 1$ then Lyapunov spectrum is $\{+,-\}$ 


\section{Table of results for Lyapunov spectra}

We present our results in the form of Table 1.

\begin{tabular}{|c|c|c|c|}
\hline & $\frac{2 g l}{a^{2} \gamma^{2}}<1$ & $\frac{2 g l}{a^{2} \gamma^{2}}=1$ & $\frac{2 g l}{a^{2} \gamma^{2}}>1$ \\
\hline \multirow{5}{*}{$\begin{array}{l}\text { Horizontal } \\
\text { modulation }\end{array}$} & $(0,0)$ & $(0,0)$ & $(0,0)$ \\
\hline & $\{+,-\}$ & $\{0,0\}$ & $\{0,0\}$ \\
\hline & $(\pi, 0)$ & $(\pi, 0)$ & $(\pi, 0)$ \\
\hline & $\{+,-\}$ & $\{+,-\}$ & $\{+,-\}$ \\
\hline & $\begin{array}{c}\left(\arccos \left(\frac{2 g l}{a^{2} \gamma^{2}}\right), 0\right) \\
\{0,0\}\end{array}$ & $\begin{array}{c}\left(\arccos \left(\frac{2 g l}{a^{2} \gamma^{2}}\right), 0\right) \\
\{0,0\}\end{array}$ & \\
\hline \multirow{5}{*}{$\begin{array}{c}\text { Vertical } \\
\text { modulation }\end{array}$} & $(0,0)$ & $(0,0)$ & $(0,0)$ \\
\hline & $\{0,0\}$ & $\{0,0\}$ & $\{0,0\}$ \\
\hline & $(\pi, 0)$ & $(\pi, 0)$ & $(\pi, 0)$ \\
\hline & $\{0,0\}$ & $\{0,0\}$ & $\{+,-\}$ \\
\hline & $\begin{array}{c}\left(\arccos \left(-\frac{2 g l}{a^{2} \gamma^{2}}\right), 0\right) \\
\{+,-\}\end{array}$ & $\begin{array}{c}\left(\arccos \left(-\frac{2 g l}{a^{2} \gamma^{2}}\right), 0\right) \\
\{0,0\}\end{array}$ & \\
\hline \multirow{3}{*}{$\begin{array}{c}\text { Circular } \\
\text { modulation }\end{array}$} & $(0,0)$ & $(0,0)$ & $(0,0)$ \\
\hline & $\{0,0\}$ & $\{0,0\}$ & $\{0,0\}$ \\
\hline & $\begin{array}{c}(\pi, 0) \\
\{+,-\}\end{array}$ & $\begin{array}{c}(\pi, 0) \\
\{+,-\}\end{array}$ & $\begin{array}{c}(\pi, 0) \\
\{+,-\}\end{array}$ \\
\hline
\end{tabular}

\section{Numerical simulations}

We support our conclusion by numerical simulations for several typical cases. On Fig.1-5 the phase portraits for all three cases, the horizontal, vertical and circular modulation are presented in the neighbourhood of the points $(0,0)$ and $(\pi, 0)$. We can observe the conversion of the fixed point from focus to the saddle point for the reasonably small decay $\epsilon$, the real trajectories for the averaged systems. 

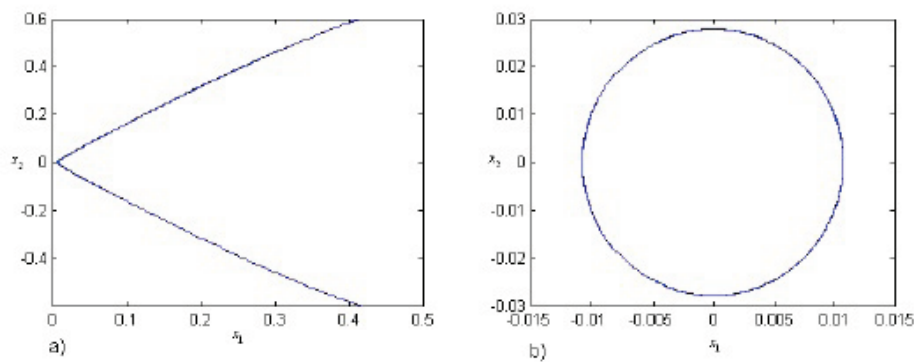

Figure 1. Horizontal modulation at $(0,0)$ : (a) for the case $\frac{2 g \ell}{a^{2} \gamma^{2}}<$ 1 where $a=10, \gamma=0.5, g=9.8, \ell=1$ (b) for the case $\frac{2 g \ell}{a^{2} \gamma^{2}}>1$ where $a=5, \gamma=0.5, g=9.8, \ell=1$
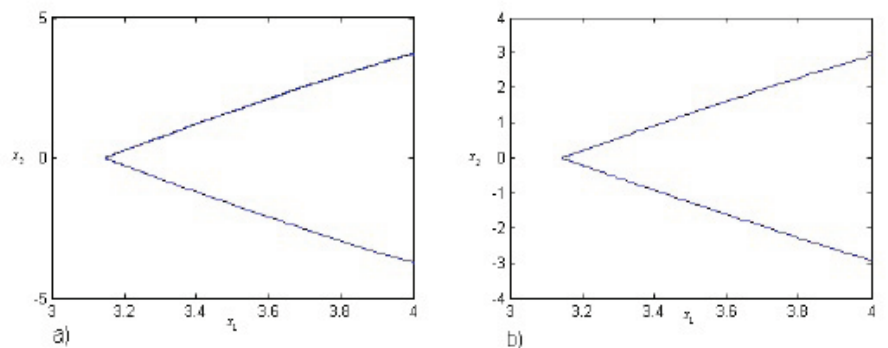

Figure 2. Horizontal modulation at $(\pi, 0)$ : (a) for the case $\frac{2 g \ell}{a^{2} \gamma^{2}}<$ 1 where $a=10, \gamma=0.5, g=9.8, \ell=1$ (b) for the case $\frac{2 g \ell}{a^{2} \gamma^{2}}>1$ where $a=5, \gamma=0.5, g=9.8, \ell=1$ 

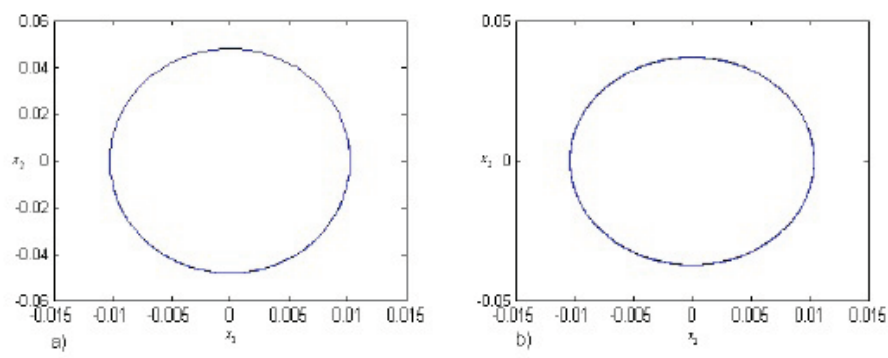

FiguRE 3. Vertical modulation at $(0,0)$ : (a) for the case $\frac{2 g \ell}{a^{2} \gamma^{2}}<1$ where $a=10, \gamma=0.5, g=9.8, \ell=1$ (b) for the case $\frac{2 g \ell}{a^{2} \gamma^{2}}>1$ where $a=5, \gamma=0.5, g=9.8, \ell=1$
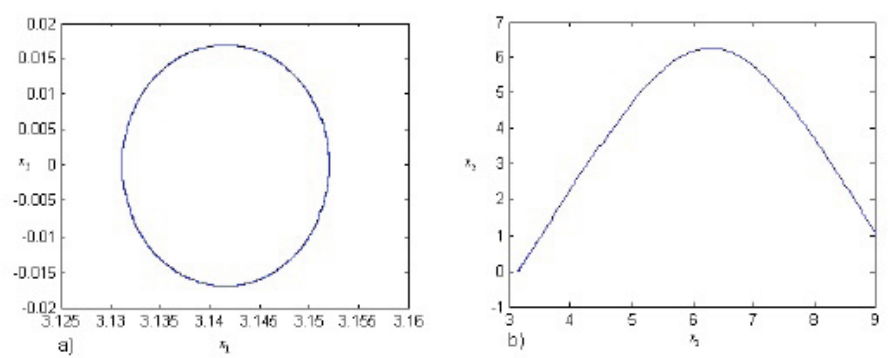

FIGURE 4. Vertical modulation at $(\pi, 0)$ : (a) for the case $\frac{2 g \ell}{a^{2} \gamma^{2}}<1$ where $a=10, \gamma=0.5, g=9.8, \ell=1$ (b) for the case $\frac{2 g \ell}{a^{2} \gamma^{2}}>1$ where $a=5, \gamma=0.5, g=9.8, \ell=1$

\section{Comparison and Conclusion}

Kapitza averaging application changes drastically the equilibrium properties of nonlinear oscillator. It can be demonstrated by calculation of Lyapunov spectra in the neighbourhood of the stable points for Kapitza oscillator. From the table in all three cases we have the conversion of Lyapunove spectrum from the focus to the saddle point.On comparison we observe that the system for horizontal oscillation becomes unstable at origin if $2 g \ell / a^{2} \gamma^{2}<1$ while for vertical and circular oscillations of pivot,the system remains stable at origin. Also we observe that the system remains stable for vertical oscillation at each of the points under the condition $2 g \ell / a^{2} \gamma^{2}=1$ but for horizontal modulation it becomes unstable at $(\pi, 0)$. At non-trivial points, the system is always stable for horizontal modulation but it becomes unstable at non-trivial point for vertical modulation under the condition 

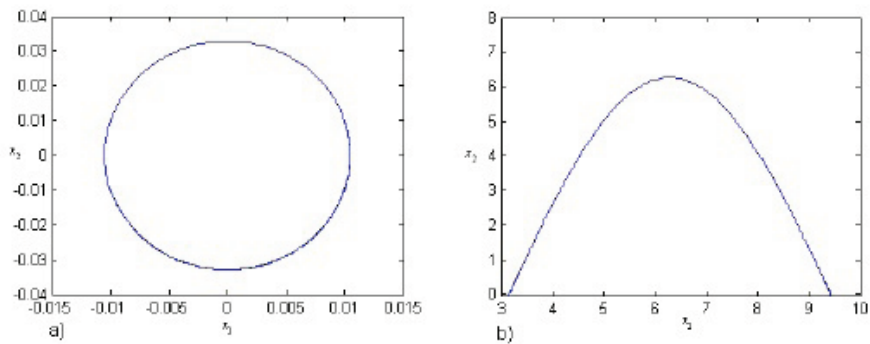

Figure 5. Circular modulation at $(\pi, 0)$ : (a) At $(0,0)$ for the case $\frac{2 g \ell}{a^{2} \gamma^{2}} \lesseqgtr 1$ where $a=10, \gamma=0.5, g=9.8, \ell=1$ (b) At $(\pi, 0)$ for the case $\frac{2 g \ell}{a^{2} \gamma^{2}} \lesseqgtr 1$ where $a=5, \gamma=0.5, g=9.8, \ell=1$

$2 g \ell / a^{2} \gamma^{2}<1$. The dimensional less parameter $2 g \ell / a^{2} \gamma^{2}$ does not affect the stability of the system at each of its stable points for oscillation of pivot along a vertical circle.Our results can be easily extended for the case of non-harmonic modulation.

Acknowledgement: The authors wish to thank Dr. Sergei Borisenok for valuable suggestions.

\section{References}

[1] Baker, G.L. and Gollub, J.P., Chaotic Dynamics, Cambridge University Press, Cambridge, 1990.

[2] Brown, L.S., "Quantum motion in a Paul trap", Phys. Rev. Lett., 66 (1991), 527-529.

[3] Bullo, F. "Averaging and vibrational control of mechanical systems", SIAM J. Control Optim. 41 (2002), 542-562.

[4] Gilary, I., Moiseyev, N., Rahav, S., and Fishman, S. "Trapping of particles by lasers: the quantum Kapitza pendulum", J.Phys. A, Math. Gen., 36 (2003), L409-L415.

[5] Kapitza, P. L., "Dynamic stability of a pendulum with an oscillating point of suspension", Journal of Experimental and Theoretical Physics, 21:5 (1951), 588-597.

[6] Landau, L.D. and Lifshitz, E.M., Mechanics, Pergamon Press, Oxford, 1960.

[7] Levi M., and Weckesser, W. "Stabilization of the inverted pendulum by high frequency vibrations", SIAM J., Mathe. Anal., 35 (2003), 844-867.

[8] Levi, M. "Stability of inverted pendulum- a topological explanation", SIAM Rev., 30 (1988), 639-644.

[9] Nakamura, Y., Suzuki, T., and Koimura, M. "Nonlinear behavior and control of a nonholonomic free-joint manipulator", IEEE Trans. Robot. Autom., 13 (1997), 853-862.

[10] Paul, W., "Electromagnetic traps for charged and neitral particles", Rev. Mod. Phys., 62 (1991), 527-529.

[11] Robinson, R.C., An Introduction to Dynamical Systems: Continuous and Discrete, Pearson Education, Inc, New Jersey, 2004. 